\title{
Upgrading the Gemini Planet Imager calibration unit with a photon counting focal plane wavefront sensor
}

Marois, Christian, Gerard, B., Lardière, O., Anthony, A., Bradley, C., et al.

Christian Marois, B. Gerard, O. Lardière, A. Anthony, C. Bradley, J. Dunn, Q. Fu, T. Hardy, W. Heidrich, G. Herriot, E. Nielsen, S. Sivanandam, D. Sivransky, S. Thibault, W. Thompson, J.-P. Véran, "Upgrading the Gemini Planet Imager calibration unit with a photon counting focal plane wavefront sensor," Proc. SPIE 11448, Adaptive Optics Systems VII, 1144873 (14 December 2020); doi: 10.1117/12.2563010

SPIE. Event: SPIE Astronomical Telescopes + Instrumentation, 2020, Online Only 


\title{
Upgrading the Gemini Planet Imager calibration unit with a photon counting focal plane wavefront sensor
}

Christian Marois*a,b, B. Gerard ${ }^{\mathrm{c}}$, O. Lardière ${ }^{\mathrm{a}}$, A. Anthony ${ }^{\mathrm{a}}$, C. Bradley ${ }^{\mathrm{b}}, \mathrm{J}_{\text {. Dunn }}^{\mathrm{a}}, \mathrm{Q}^{\mathrm{Q}}$. Fu ${ }^{\mathrm{d}}, \mathrm{T}$. Hardy $^{\mathrm{a}}$, W. Heidrich ${ }^{\mathrm{d}}$, G. Herriot ${ }^{\mathrm{a}}$, E. Nielsen ${ }^{\mathrm{e}}$, S. Sivanandam ${ }^{\mathrm{f}}$, D. Sivransky ${ }^{\mathrm{g}}$, S. Thibault ${ }^{\mathrm{h}}$, W. Thompson $^{\mathrm{a}, \mathrm{b}}$, J.-P. Véran ${ }^{\mathrm{a}}$ and the GPI CAL2.0 team

aNational Research Council of Canada Herzberg Astronomy and Astrophysics, 5071 W Saanich Rd, Victoria, BC V9E 2E7 Canada; ${ }^{b}$ University of Victoria, 3800 Finnerty Road, Victoria, BC V8P 5C2 Canada; 'University of California Santa Cruz, 1156 High St, Santa Cruz, CA 95064, USA; ${ }^{\text {King }}$ Abdullah University of Science and Technology 4700 King Abdullah University of Science and Technology Thuwal 23955-6900 Kingdom of Saudi Arabia; New Mexico State University, P.O. Box 30001, MSC 4500, Las Cruces, NM 88003, USA; fUniversity of Toronto, 27 King's College Circle, Toronto, ON M5S 1A1 Canada; 'Cornell University, 616 Thurston Ave. Ithaca, NY 14853 USA; hUniversité Laval, 2325 Rue de l'Université, Québec, QC G1V 0A6 Canada

\begin{abstract}
High-contrast imaging instruments have advanced techniques to improve contrast, but they remain limited by uncorrected stellar speckles, often lacking a "second stage" correction to complement the Adaptive Optics (AO) correction. We are implementing a new second stage speckle-correction solution for the Gemini Planet Imager (GPI), replacing the instrument calibration unit (CAL) with the Fast Atmospheric Self coherent camera Technique (FAST), a new version of the self-coherent camera (SCC) concept. Our proposed upgrade (CAL2.0) will use a common-path interferometer design to enable speckle correction, through post-processing and/or by a feedback loop to the AO deformable mirror. FAST utilizes a new type of coronagraphic mask that will enable, for the first time, speckle correction down to millisecond timescales. The system's main goal is to improve the contrast by up to 100x in a halfdark hole to enable a new regime of science discoveries. Our team has been developing this new technology at the NRC's Extreme Wavefront control for Exoplanet and Adaptive optics Research Topics (NEW EARTH) laboratory over the past several years. The GPI CAL2.0 update is funded (November 2020), and the system's first light is expected late 2023.
\end{abstract}

Keywords: Adaptive optics, imaging, near infrared, focal plane wavefront sensing, brown dwarfs, debris disks, exoplanets

\section{INTRODUCTION}

High-contrast direct imaging observations have enabled a plethora of new science cases over the past two decades, including the chemistry and dynamics of young, self-luminous giant exoplanet atmospheres (e.g., [1]), understanding giant planet formation in action and its connection to circumstellar disks (e.g., [2]), and the occurrence rates of wide orbit, giant exoplanets (e.g., [3]).

Despite these ground-breaking results coming from direct imaging, surveys and non-detections around hundreds of nearby stars have revealed that wide orbit giant exoplanets are rare. The most sensitive-to-date results [3] have shown that only 9\% of stars host exoplanets between 5-13 times the mass of Jupiter, or $\mathrm{M}_{\mathrm{Jup}}$, and 10-100 AU, whereas radial velocity (RV) surveys, which are more sensitive to older, lower-mass exoplanets at separations within 10 AU but cannot obtain exoplanet spectra, have found that giant exoplanets within $10 \mathrm{AU}$ and below $5 \mathrm{M}_{\mathrm{Jup}}$ are more abundant, up to about 30\% [4]. Therefore, there is a clear motivation for future exoplanet imaging instruments and surveys to push further into new areas of parameter space, including lower masses, closer separations, and older ages. The main limit for the direct imaging technique is the residual "speckle" noise in the image of a high contrast imaging instrument, masquerading as the exoplanet and preventing initial detection in the recorded "raw" images. These speckles originate from a combination of (1) residuals after an adaptive optics (AO) system has made an initial correction of the optical aberrations caused by atmospheric turbulence, and (2) imperfections in the polishing and reflectivity of individual

Adaptive Optics Systems VII, edited by Laura Schreiber, Dirk Schmidt, Elise Vernet, Proc. of SPIE

Vol. $11448,1144873 \cdot$ @ C 2020 SPIE · CCC code: $0277-786 X / 20 / \$ 21 \cdot$ doi: $10.1117 / 12.2563010$ 
optical surfaces from the telescope and within the instrument. Furthermore, these speckles are neither static nor achromatic, causing an evolution of the speckle pattern over time and wavelength, respectively.

Speckle noise that is both quasi-static (throughout the image) and dynamic (closer to the star and stronger in one direction, related to the wind direction across the telescope aperture), and chromatic (i.e., evolving with wavelength) is what currently limits the achievable final contrast (of order $10^{-6}$ after post-processing, indicating how much fainter a planet relative to the star would be detectable). Two main strategies are usually used to further suppress speckle noise:

1. spectral differential imaging (SDI; [5]), distinguishing diffraction-induced radially moving speckles as a function of wavelength from an unmoving exoplanet, and

2. angular differential imaging (ADI; [6]), distinguishing unmoving speckles as a function of time from an azimuthally moving exoplanet by adjusting the instrument rotator to track the telescope pupil instead of the sky (exoplanet motion being generated by the Earth's rotation).

Although these strategies are combined with advanced least-squares subtraction algorithms (e.g., [7]), SDI and ADI remain fundamentally limited by wavelength- and time-dependent speckle evolution (i.e., "chromaticity" and "stability", respectively); $\mathrm{AO}$ residuals cause an uncorrelated "halo" between subsequent long exposure frames that limit the achievable contrasts with ADI (e.g., [8]). Fresnel propagation effects cause chromatic aberrations (e.g., [9]), which limit SDI. Flexure and temperature-related changes over time (e.g., [10]) also further limit ADI. Thus, new speckle subtraction methods are needed to help detect exoplanets that are colder, closer in, and/or lower in mass.

The past 15 years have seen important progress in the development of focal plane wavefront sensing techniques, where the science camera is used to both sense aberrations and perform science. From the pioneer work of Guyon 2004 [11] where coherence was proposed to measure the focal plane electric field in high-contrast imaging, to set fundamental limits when combining it with active deformable corrections (Borde \& Traub 2006, [12]), many approaches have been proposed and tested since then in laboratory settings and some on sky. One technique, the self-coherent camera (SCC, [13]), consists of using a dual aperture Lyot stop where the stellar light being diffracted outside the pupil by the focal plane mask goes in both apertures, while the off-axis exoplanet planet light only goes through one. In this concept, all the stellar speckle noise becomes fringed and these can be used to measure the focal plane electric field. A new postprocessing technique, called coherent differential imaging (CDI), uses coherent starlight in the focal plane to measure and to subtract its own optical aberrations without removing any incoherent exoplanet light. CDI is able to (1) subtract speckles on timescales faster than evolving quasi-static and AO residuals (which is not possible with ADI), and (2) subtract speckles at each wavelength without relying on images acquired at other wavelengths to mitigate chromaticity (which is not possible with SDI). CDI post-processing can, in theory, be also applied with a deformable mirror control loop to remove residual noise from imperfect deformable mirror corrections, from lag errors, or for regions outside the deformable mirror control area. One of the main limitations of the SCC concept is the limited amount of light going in the second small Lyot stop pinhole, requiring long integrations to detect fringes in the focal plane. A recent modification to the SCC concept, called FAST [14], boost the pinhole signal by up to a million, having the potential to show fringes on ms timescale on bright stars. The new method uses a specialized focal plane mask to take the stellar spread function core light and focus it on the Lyot stop pinhole. Our project consists of building a FAST CDI-enabled system for the Gemini Planet Imager (GPI, [15]) high-contrast imaging instrument.

The GPI is designed primarily to directly image exoplanets and characterize them via spectroscopy. It has been installed on the Gemini South 8-m telescope in Chile since late 2013. The instrument is equipped with a high-performance AO system to correct for distortions from atmospheric turbulence, a coronagraph to attenuate the light from the star while transmitting the exoplanet light, a wavefront sensor (WFS) downstream of the AO and coronagraph systems to measure remaining aberrations left uncorrected by the AO system, called the CAL, and lastly an integral field spectrograph (IFS). GPI will soon (early 2021) be moved from the Gemini South telescope to the twin 8-m Gemini North telescope in Hawaii, during which the AO and IFS boxes will be upgraded (hereafter referred to as "GPI2.0"), modifying the AO system to a pyramid wavefront sensor (PWFS) [16] to enable access to fainter stars, the coronagraph apodizer (on the AO bench) to improve diffraction suppression and robustness to low order aberrations, and the IFS to include a broadband mode that will enable spectral characterization over a broader wavelength range than before. Our new approved project will replace the CAL system with a FAST-enable camera, along with other focal plane wavefront sensing methods. The main goals of scientific operations for this upgrade, hereafter referred to as CAL2.0, are: 
1. Improve the current GPI IFS raw contrast by up to a factor of $20 \mathrm{x}$ by correcting the remaining residual atmospheric and quasi-static errors using the AO-based DM control. The main goal is to enable new science cases for the IFS instrument, while keeping the same core GPI science capabilities (i.e., spectroscopy and polarization) with the same field-of-view.

2. Enable new science cases by using the CAL2.0 FAST SCC camera as a narrow band imager, including filters at $0.84 \mathrm{um}, \mathrm{Y}, \mathrm{J}$, and $\mathrm{H}$ bands, by reaching up to $100 \mathrm{x}$ better than the current GPI integral field spectrograph (IFS) final contrasts (using DM control and CDI post-processing). Imaging modes will include a few narrowband filters per bandpass to allow detection and some level of basic characterization.

\section{CAL2.0 SCIENCE CASES}

FAST will enable new science cases, both for current 8 m-class telescopes (i.e., GPI) and future $30 \mathrm{~m}$-class Giant Segmented Mirror Telescopes (GSMTs). In Fig. 1, simulations show that CAL2.0 will enable direct images of indirectly detected exoplanets.

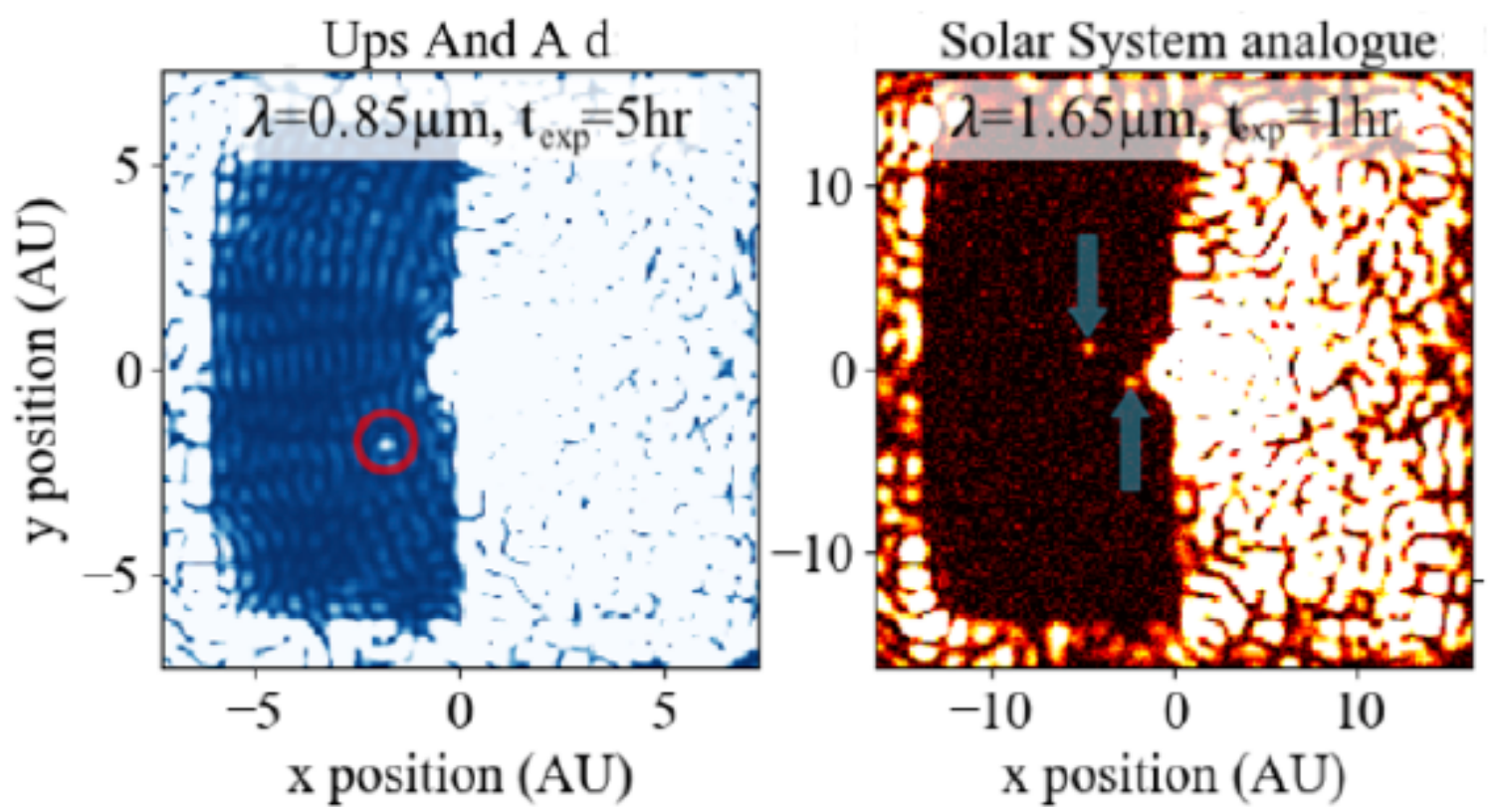

Fig. 1: Simulated examples of science enabled by the CAL2.0 for GPI [17]. Left: reflected light imaging of the radial velocity exoplanet Ups And A d (10 Jupiter masses, 2.5 AU semi-major axis at $13.5 \mathrm{pc}$ ). Right: thermal imaging of two Jupiter mass planets at 5 and $10 \mathrm{AU}$ orbiting a $30 \mathrm{Myr}$ old star at $30 \mathrm{pc}$.

Although host star radial velocities have revealed a plethora of self-luminous giant exoplanets and measured their masses, directly imaging their reflected starlight would provide an atmospheric characterization and crucial dynamical mass calibration (since the dynamical mass cannot be inferred through imaging alone). This synergy and improved calibration will also further increase with new detections from the Gaia satellite via astrometry ([18]; i.e., detecting exoplanets via the reflex motion of the host star by measuring its position on the sky over time) that can also similarly be directly imaged (both for young stars in thermal emission and old stars in reflected light); such calibrations would provide a direct window into measuring the mass-luminosity relation for young gas giants to resolve existing discrepancies between cooling curves used thus far in the direct imaging field to indirectly measure masses. CAL2.0 will also detect thermal emission from young self-luminous exoplanets below $1 \mathrm{M}_{\mathrm{Jup}}$ at $1 \mathrm{AU}$, enabling a huge advancement in survey capabilities as illustrated in Fig. 2. 

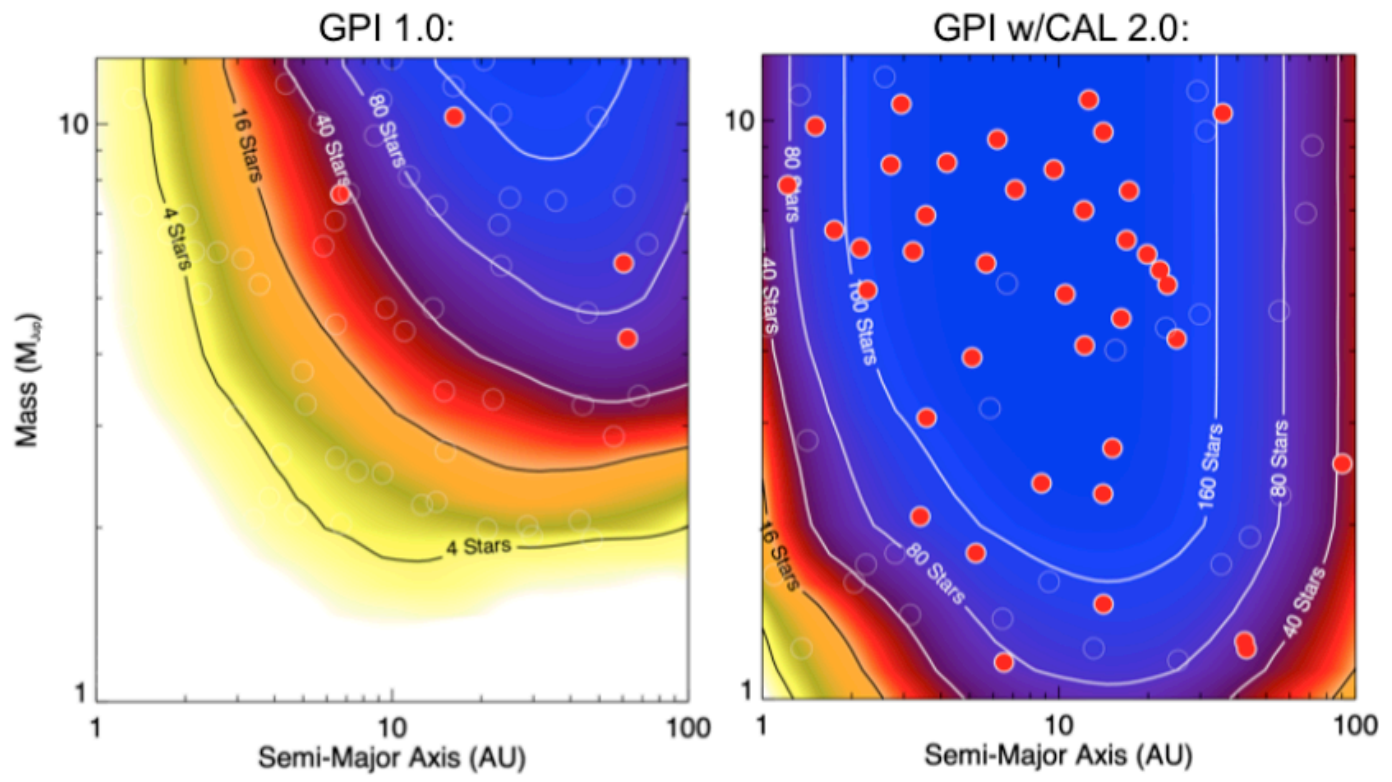

Fig. 2: Left panel, exoplanet sensitivity and detections for a 300-star survey using GPI1.0 and (right) for CAL2.0 as a science camera. Detected planets in the sample are shown as red-filled circles, while undetected planets are shown as open white circles. The new CAL system is expected to increase the sensitivity to lower mass exoplanets at smaller separations.

Since current direct imaging surveys are not yet sensitive to these Solar System scales [3], CAL2.0 will provide the first estimates of the prevalence of true Jupiter analogues beyond our Solar System (i.e., a region of parameter space for which RV surveys are also not yet sensitive; [4]), enabling a plethora of new detections. Fig. 2 illustrates that a 300-star survey with CAL2.0 is expected to detect 39 exoplanets, whereas GPI1.0 (i.e., the current GPI) would only expect four exoplanets (also consistent with the four systems around which exoplanets were detected in the GPI Exoplanet Survey (GPIES; [3]).

Accordingly, CAL2.0 will also serve as a crucial technology demonstration for the tolerances and requirements needed for future reflected light imaging of habitable exoplanets on GSMTs. As a result of the tests and calibrations with giant exoplanets using CAL2.0, Fig. 3 shows that once GSMTs, such as TMT, are available in the late 2020s, FAST will enable the direct detection of exoplanets in the habitable zone around the nearest stars.

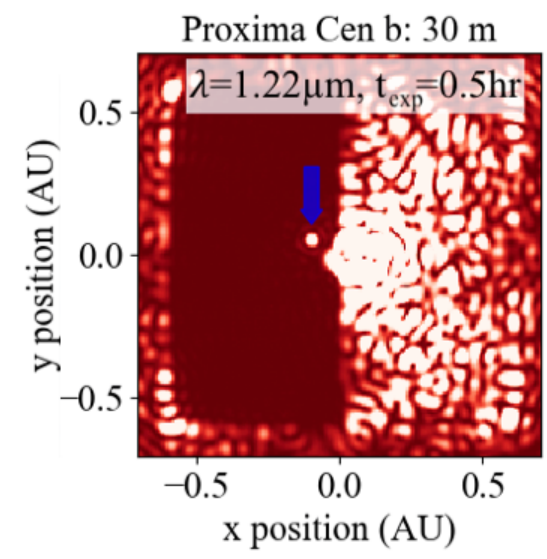

Fig. 3. Habitable exoplanet imaging for future 30-m class extremely large telescopes [19], simulating the radial velocity exoplanet Proxima Cen b (1 Earth mass in the habitable zone at $1.3 \mathrm{pc} ;[20])$. 
In addition to the above capabilities of CAL2.0 as a science instrument, improved common-path wavefront control will also greatly improve the image quality at the GPI IFS. A reduction in speckle noise at the smallest angular separations will further improve sensitivity to spectroscopy of self-luminous exoplanets located at the peak of the period distribution suggested by previous radial velocity surveys (e.g., [4]). A suppressed and more stable speckle field will allow for a more effective post-processing speckle subtraction of IFS data, removing a significant source of systematic error when measuring the spectra of these objects. Errors like these, that are correlated in neighboring spectral channels, can lead to significant discrepancies between spectra of the same object obtained on different epochs or with different instruments (e.g, [21], [22]), and even to the incorrect categorization of a distant background star as a planetary-mass companion [23]. CAL2.0 will thus enable more accurate and precise determinations of the atmospheric properties of substellar companions with the GPI IFS. K-band characterization, although not included in the CAL2.0 design due to the limited gains expected from simulated contrast curves and the greater complexity in design requirements, will still be enabled by the IFS, enhanced by the CAL2.0 H band correction.

CAL2.0 will also enable new Solar System science. Moons of giant planets (Jupiter, Saturn, Uranus, and Neptune) and large main-belt asteroids (MBAs) are mini-geological worlds by themselves with complex interactions due to the presence of the massive planet and other satellites. Deriving the shape and surface properties of large asteroids as well as understanding variable phenomena, such as the volcanoes of Io, the geysers on the south pole of Enceladus, the climate of Titan, and the gardening effect on the radiated icy surface of other satellites, requires long term monitoring that can be provided only from 8-m class ground-based telescopes equipped with high angular resolution imaging (i.e., extreme AO). Several icy satellites will be the targets of upcoming space missions (NASA Europa Clipper and ESA Juice) reinforcing the need for ground-based monitoring during this coming decade. Main satellites of the giant planets and $\sim 100$ MBAs are directly observable with CAL2.0 considering a limit in AO guide star magnitude of 12 and their small apparent size (<2"). Non-coronagraphic imaging with the CAL2.0 at $\sim 0.85 \mu \mathrm{m}$ and high strehl ratio ( $>60 \%)$ with an angular resolution of 10 mas is a unique capability to detect features on the surface, such as craters and deposits on large asteroids as well as surface changes on icy satellites or hot component volcanoes on Io.

The existing GPI instrument has delivered a significant amount of circumstellar disk science, producing resolved imaging of disk structures within 1 arcsecond of their host stars [24]. The polarization imaging capabilities of GPI provided a means of superior stellar subtraction for disks, due to the fact that disk emission is inherently polarized while stellar emission is not. GPI2.0 will still be able to take advantage of the polarization capabilities with the GPI IFS but with additional frontend capability for CAL2.0 imaging of disks, enabling better recovery of non-polarized (i.e., total intensity) emission of disks, particularly face-on disks (i.e., CDI processing, unlike ADI processing, is agnostic to disk inclination angle). Such detections will enable estimates of the polarization fraction and hence tighter constraints on grain composition and shape, as well as the removal of bias in the samples of disk-hosting stars that can be targeted. Most enticingly, the key signatures in disks associated with planets are best observed in disks in which azimuthal and radial structure can be resolved, i.e., closer to face-on disks.

As Dong et al. (2015; [25]) showed, spiral structure in disks can place constraints on the position and mass of exoplanets that are too low mass to be directly detected. Recent state-of-the-art high contrast instruments have also directly detected

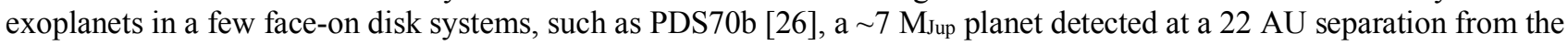
star. Improved face-on disk imaging and deeper achievable contrasts with CAL2.0 should help to detect more systems like PDS 70b, combining the imaging of planets and their associated disks, both in formation and at later stages in debris disks.

Characterizing young stellar binaries will also be greatly aided by both the coronagraphic and non-coronagraphic $0.85 \mu \mathrm{m}$ imaging modes of CAL2.0. Solar-type stars take tens of Myr to reach the main sequence, moving along the color-magnitude diagram, quickly down the Hayashi track and then across the Henyey track. As a result, the ages of young moving groups (e.g. the $24 \mathrm{Myr}$ beta Pic moving group, which hosts two directly imaged exoplanets, many brown dwarfs, and multiple circumstellar disks) can be constrained by precisely determining the location of moving group stars in their evolutionary sequences. In particular, close binaries, where dynamical masses can be measured from a combination of radial velocities and the visual orbit, and resolved photometry of both components can be obtained, place very tight age constraints on the entire moving group (e.g. [27]). Previously the imaging component of such studies usually had to be done in the NIR, but CAL2.0 would allow these observations to be done in the visible. The shorter 
wavelength provides better resolution, and better precision in derived ages, since visible magnitude changes more quickly as a function of mass compared to the NIR. The best binaries in the nearby moving groups are at separations $<100$ mas, too close for speckle imaging, while non-redundant masking produces degeneracies between separation and flux ratio. Direct imaging with CAL2.0 will enable direct measurements of flux ratio, separation, and position angles of new binaries too close for current systems, at a wavelength range most useful for constraining ages.

A few other areas of study will be enabled via the enhanced capabilities of CAL2.0, including the outflows from evolved stars and the innermost regions of nearby active galactic nuclei (AGN). The last phases of a star's life, in which significant mass loss occurs, are poorly understood, but play an essential role in understanding both stellar and Galactic evolution through stellar population synthesis models. Key missing observations of evolved stars are those at tight angular separations $(<2$ ") to study outflows and dust formation, which are needed to provide crucial feedback for stellar evolutionary models. Since these stars are generally very bright, they are ideal targets for CAL2.0, which will provide significantly enhanced contrast. Improved performance on these sources are required in order to distinguish the faint outflow signatures from the PSF speckle noise that has plagued observations with GPI1.0. Furthermore, a combination of increased limiting magnitude through the new PWFS detector in the AO upgrade and increased contrast with CAL2.0 is key to providing extragalactic science opportunities with GPI for the first time. Polarimetry mode IFS observations, fed by the enhanced speckle correction enabled by CAL2.0, of bright AGN probe the inner $100 \mathrm{pc}$ to the black holes and actually allow for studies of the magnetic fields that launch the jets from the AGN (e.g., [28]). Some of the brightest nearby AGN are easily or only observable from the Northern Hemisphere, such as NGC 4151 and 3C 273.

\section{CAL 2.0}

The original GPI CAL unit was designed to measure only for the quasi-static noise component and was never fully operational mainly due to vibrations. The main CAL2.0 architecture of our proposed upgrade is illustrated in Fig. 4, comparing the existing CAL sub-system (CAL1.0) with our upgrade (CAL2.0).

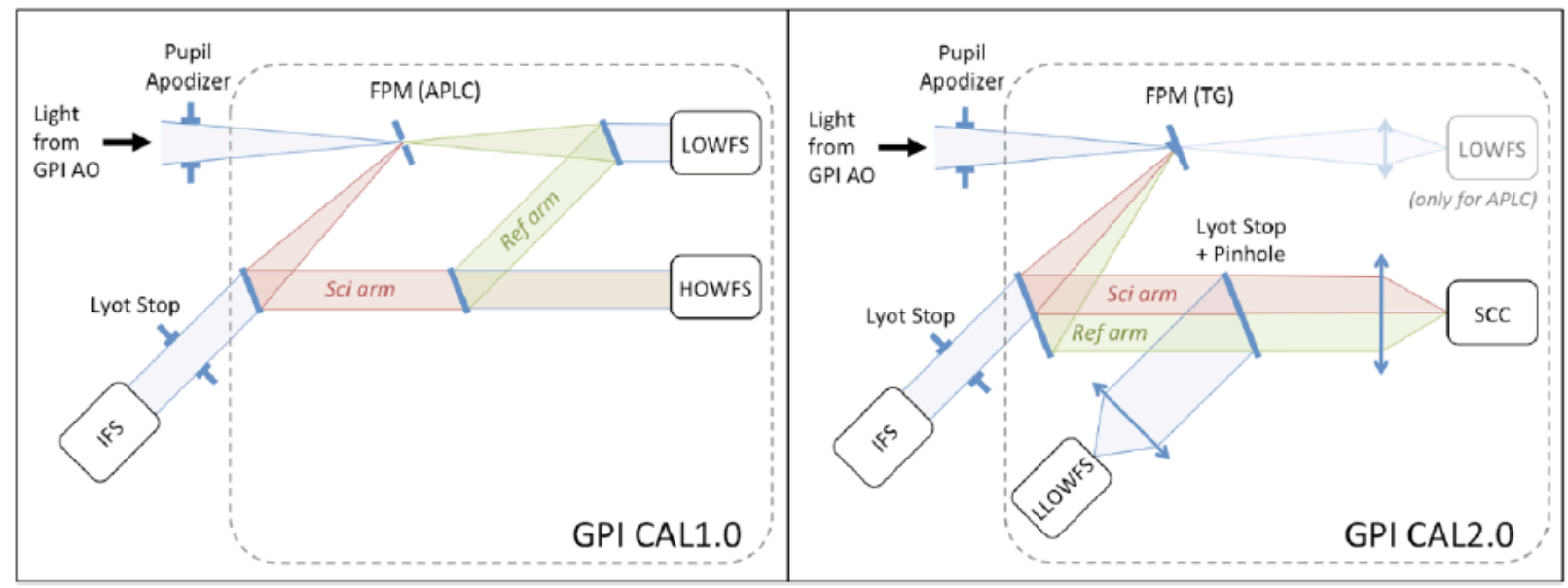

Fig. 4: Schematic view of our CAL 2.0 upgrades (right) compared to the CAL1.0 subsystem (left).

Upstream of the CAL unit (not shown in Fig. 4), light from the telescope or artificial light source propagates through a series of tip/tilt and DM wavefront correctors. A dichroic then sends the visible light to the PWFS [16] and the near infrared (NIR) light further downstream. We will install a new dichroic with a cut-off wavelength of $0.84 \mu \mathrm{m}$ to enable a new shorter wavelength imaging mode for exoplanet reflected light imaging and solar system science. Downstream NIR light (now shown in Fig. 4) is then modified by a custom pupil plane apodizer mask and Tip/tilt Gaussian (TG) coronagraphic focal plane mask (FPM), see Fig 5. 

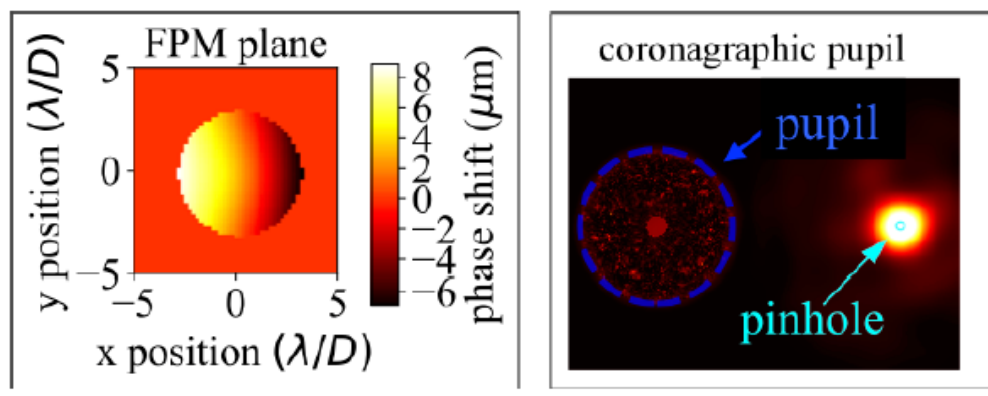

Fig. 5: Left: The Tip/tilt+Gausian (TG) Focal Plane Mask (FPM), the core new technology enabling FAST. Right: the pupil plane downstream of this custom FPM generates an off-axis pupil at the location of the pinhole in the SCC's interferometer mask, boosting the intensity transmitted through this pinhole by 6 orders of magnitude. Both panels are adapted from simulations in [14].

A Beam Splitter (BS) then splits the light between the IFS and CAL paths. The CAL path includes a custom interferometer mask (the Lyot stop), optics, and dedicated fast IR detectors, including both a low order WFS (LOWFS) and high order WFS (HOWFS; i.e., the FAST for CAL2.0). Generically, a LOWFS is used to align the PSF on the FPM for low order modes, such as tip, tilt, and focus, at the same wavelength as the science image to avoid wavelength dependent aberration effects (which in the case of GPI is at NIR wavelengths, distinct from the visible light PWFS), whereas a HOWFS is designed to measure mid- to high-order modes that remain uncorrected after the initial AO correction. The classical LOWFS design in the CAL1.0 is no longer possible with our custom TG FPM, which now redirects the PSF core downstream of the FPM to be alongside the science beam instead of being sent through a pinhole in the FPM as in the CAL1.0. Thus, the LOWFS in our proposed upgrade is a Lyot-based LOWFS, or LLOWFS ([29], [30]), using a custom reflective Lyot stop mask to redirect light normally blocked by this mask to a separate detector for low order wavefront sensing ([30],[31]). A CoDR-level schematic view of the optical system is presented in Fig. 6. Fig 7 shows the available space inside GPI to fit the CAL2.0 box. The optical layout of the science path to the IFS remains unchanged, so the upgrade from CAL1.0 to CAL2.0 is transparent for the IFS. The two CAL Pointing-and-Centering $(\mathrm{P} \& \mathrm{C})$ mirrors and Polarization Module can be reused without modification. However, the size of the science off-axis parabola (OAP) and beam-splitter (BS) have to grow in one direction to reflect and transmit, respectively, the off-axis reference beam toward the SCC, as shown in Fig. 6. A pair of baffles are added in the science path to block the bright reference beam that could otherwise cause stray light in the IFS path by bouncing off optics' edges or mounts. 


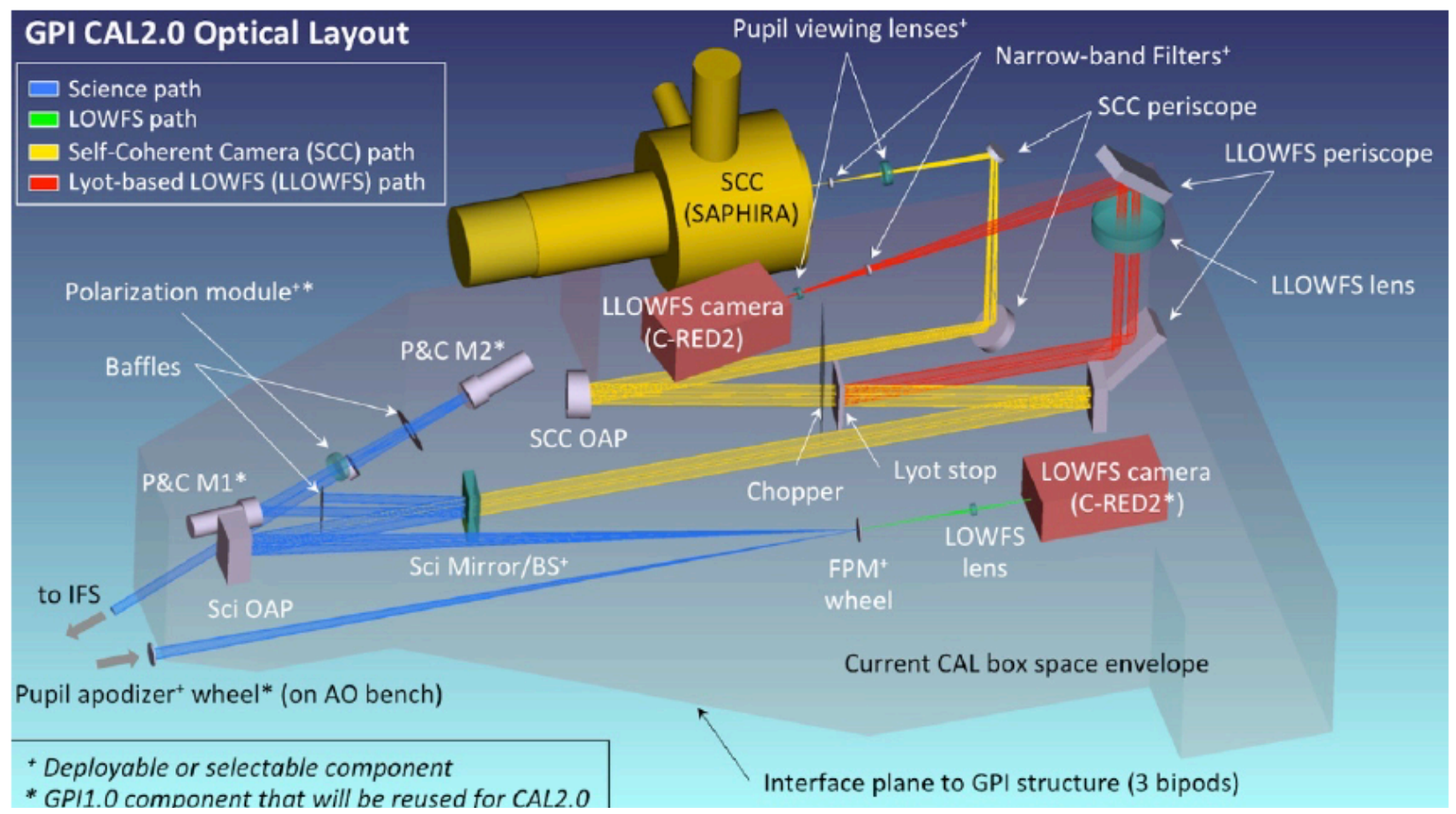

Fig. 6: Schematic view of the proposed GPI CAL2.0 subsystem. Sets of new FAST FPMs are installed in the FPM wheel to split the beam in two SCC beams, a beam-splitter reflecting part of the light toward the GPI IFS, while another fraction is propagated into the CAL2.0. A specialized two-hole SCC Lyot stop blocks the diffracted light outside the pupil, besides a small off-axis pinhole. The resulting beam is directed toward a SAPHIRA-based NRC-built camera. The reflective Lyot stop feeds a LLOWFS. To keep the old LOWFS GPI coronagraphic capability, a CRED2 is used for cases when using the GPI apodized-Lyot coronagraph (APLC) coronagraph and the PSF core light goes through the FPM.
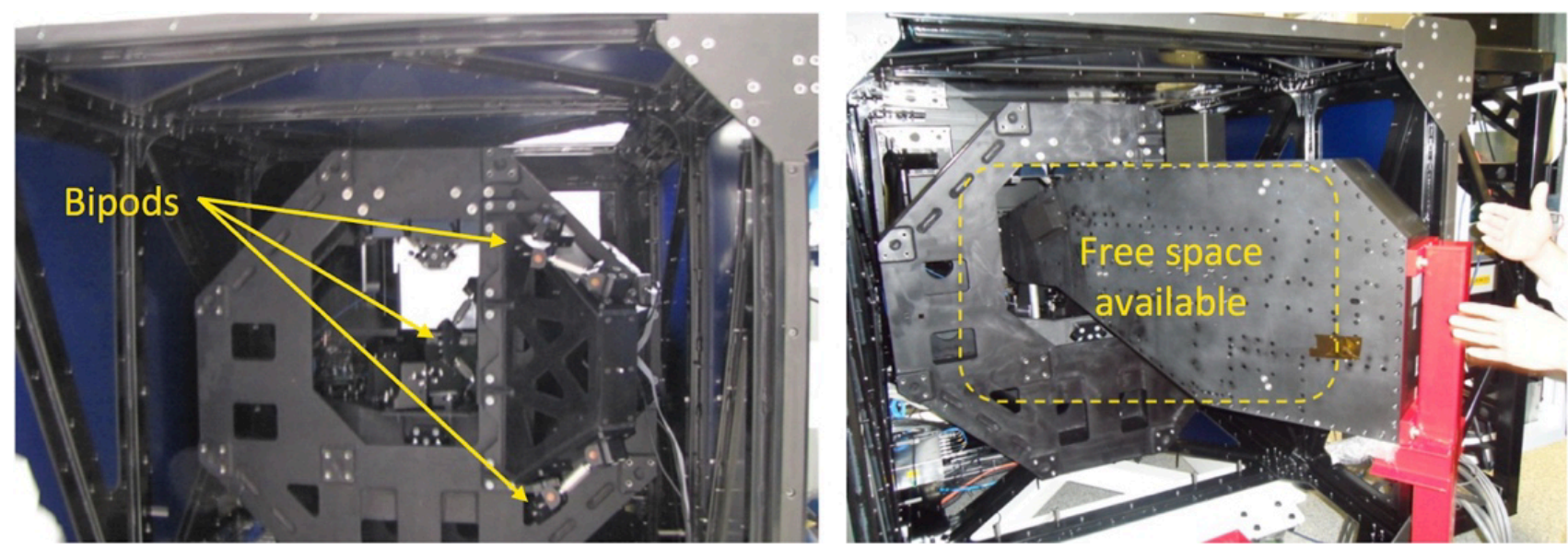

Fig. 7: Current GPI CAL box interface plane (left panel). Significant space is available on one side of the CAL box (right panel).

\section{FAST CONTROL LOOPS AND REAL TIME CONTROLLER}

The GPI real time controller (RTC) will be modified to integrate CAL2.0 functionalities. The GPI2.0 RTC is built using the Herzberg Extensible Adaptive Real-time Toolkit (HEART), a C/C++ software framework for constructing RTCs that targets general-purpose CPUs and standard networking hardware. Although HEART was originally conceived as a reconfigurable abstraction of the NFIRAOS RTC for the thirty meter telescope TMT [32], it was recognized that it could 
support not only other instruments, but also other types of AO not implemented by NFIRAOS [e.g., Ground Layer AO, Multi-Object AO, and Extreme AO] with minimal modification. Since then, HEART has evolved into a stand-alone package.

With GPI1.0, the LOWFS and CAL1.0 systems work at low speed and interface with GPI via the Top-Level Computer (TLC). With CAL2.0, where the LOWFS is functionally replaced by the LLOWFS and the HOWFS is replaced by the FAST, the speeds and frame rates are much higher, and the measurements need to be processed in real-time with minimal latency through the RTC. Therefore, the GPI2.0 RTC needs to be augmented to support these two additional WFSs. The modifications will include interfacing with the LLOWFS and SCC detectors, processing the pixels, converting the pixels to the proper modes through a matrix-vector multiplication (MVM), implementing temporal filtering, and combination/aggregation. Further customization will be needed in the GPI2.0 processing path to support the acquisition procedure.

To close the AO loop with FAST, we have to integrate both the FAST SCC and LLOWFS cameras within the GPI2.0 Real-Time Controller (RTC) running the GPI PWFS, DMs (woofer, twitter, tip/tilt stage) and AO P\&C mirrors. As these three WFSs are each in different optical paths and the LLOWFS and SCC operate in the NIR vs. the visible pyramid wavefront sensor (PWFS), NCPE and chromaticity between sensors could cause discrepant WFS measurements to "cancel out" one another. To avoid this conflict, we have designed a preliminary spatio-temporal control authority partitioning between WFSs, similar to the control-loop design for NRC-HAA's Narrow Field InfraRed Adaptive Optics System (NFIRAOS; [32]) and outlined in detail in Gerard et al. 2020 [31].

\section{ADDITIONAL FOCAL PLANE WAVEFRONT SENSING AND CONTROL METHODS}

In addition to implementing FAST-based DM control, we will also pursue additional focal plane wavefront control strategies. This multi-faceted approach will serve to (1) advance the development of these algorithms (discussed below) and (2) mitigate risk of deploying FAST. As these approaches require no additional hardware and/or RTC modification (wavefront sensing schemes and DM commands will be applied through TLC commands rather than the RTC), algorithmic development can happen in tandem of FAST hardware and software design, purchasing, and assembly.

Speckle nulling [12] was previously deployed with GP1.0 during its integration and testing (I\&T) period and then demonstrated on the fully assembled instrument, using the internal laser source and producing a factor of two reduction in average contrast over a 0.2 arcsecond annulus within the dark hole [33]. However, schedule and staffing constraints limited a full integration of speckle nulling during GPI1.0's commissioning period in Chile, and it has since not been used during regular facility operations. GPI1.0's sole coronagraphic imager-the IFS-introduced a significant constraint on the speed of applying a DM correction. The speckle nulling algorithm required nine images per iteration, which took almost 12 minutes to collect, preventing on-sky deployment. There are two primary reasons for the long data acquisition time: an integration of at least 30 seconds is required so that the generated images are not read-noise limited; additional overheads are imposed in between and after acquiring images by GPI's TLC architecture. Despite these marginal gains on GPI1.0, this prior experience has taught us important lessons. The introduction of a fast, low noise coronagraphic imager in CAL2.0 will significantly improve the operability of speckle nulling for GPI. Given the existence of a relatively mature set of speckle nulling codes tailored specifically for GPI, it makes sense to adapt these to the new coronagraphic imager introduced by CAL2.0. We propose to do so, and will fold this into a current, simultaneous effort to upgrade and modernize the entirety of the GPI scripting control library (called gpilib). In addition to the benefits mentioned at the beginning of this section, speckle nulling with the new SAPHIRA detector will provide a direct point of comparison to prior focal plane correction efforts with GPI.

Electric Field Conjugation (EFC [34]) was developed as a modification to speckle nulling to improve the efficiency of convergence in generating a dark hole. Although these two approaches are similar, EFC in principle only requires two images to converge and is thus a favorable approach to apply on-sky, while speckle nulling, as mentioned above, requires more than two images; with that said EFC also has disadvantages in that it is model-dependent, increasing the risk of systematic model-based errors, while speckle nulling is model-independent. Thus, development and analysis of both methods on the same instrument will help enable a high-fidelity comparison of the pros and cons of each. 
Phase Diversity (PD): The main benefit of implementing PD in addition to FAST is access to a larger linear range. This approach utilizes a non-linear optimizer to reconstruct the pupil plane phase over roughly up to $+/$ - a few microns peakto-valley in $\mathrm{H}$ band of linear range for low order Zernike modes [35], far greater than the $+/-400 \mathrm{~nm}$ of the SCC (Sec. 3.5.6.1 of Gerard et al. 2020b [30]). Accordingly, although GPI is designed with tens of nm rms of NCPEs, PD is a better solution to compensate for any micron rms-level differential alignment and/or quasi-static errors that may occur. Already informed by our experience with applying PD to GPI1.0 [37], a number of possible algorithmic and implementation solutions will be explored in simulation, such as the trade-offs between classical double-image vs. single image PD ([35], [36]), whether or not defocus can be applied through PWFS offsets and/or open loop woofer DM commands, and the potential benefit of utilizing the permanently defocused LLOWFS detector.

\section{TIMELINE}

The CAL2.0 project timeline and milestones are outlined in Fig. 8.

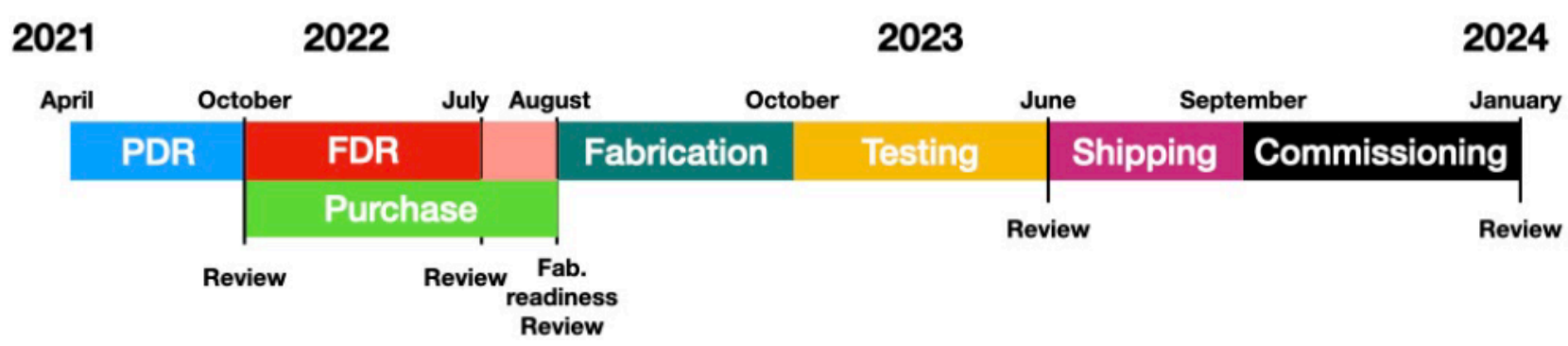

Fig. 8: The CAL 2.0 timeline. The first year is dedicated to the system's PDR and FDR. Testing is expected in the last quarter of 2022 with on sky commissioning late 2023.

The first year will consist mostly of a design phase, including optical, mechanical, electrical, and software design as well as end-to-end systems modelling that will set tolerance requirements for these designs. An external panel will review the project development after the preliminary and final designs. Long lead time items (SAPHIRA detector and OAP mirrors) will be ordered as soon as possible after the Preliminary Design Review (PDR), planned in Oct. 2022. Fabrication will be initiated shortly after the Final Design Review (FDR), and assembly thereafter as parts arrive at HAA. At the moment, after full assembly, the initial testing phase is planned to be carried out in the NEW EARTH laboratory at HAA [37], reusing existing AO-related hardware upstream of CAL2.0 (including a 468 actuator DM, visible light SH-WFS, and a coronagraphic science path already designed for NIR light).

A fully assembled CAL2.0 box in the common path of the SCC and LLOWFS detectors (i.e., including our new FPM wheel but not including the CAL PnC mirrors and polarization module) will enable systems level testing without relying on other parts of GPI to be shipped to HAA. A full in-person review is currently planned at the end of the CAL2.0 I\&T phase in June 2023. Following I\&T, CAL2.0 will be shipped to Gemini North telescope in HI, USA, where the full instrument will then be assembled, aligned, and installed on the telescope, followed by on-sky commissioning.

\section{CONCLUSIONS}

The GPI FAST-based CAL2.0 system upgrade will enable focal plane wavefront sensing at hundreds of $\mathrm{Hz}$ speed in a narrowband, send corrections to the deformable mirror (DM) to build a half dark hole, and allow CDI post-processing inside and outside the half dark hole. The CAL2.0 DM wavefront corrections will also improve the GPI IFS wavefront, allowing in-depth spectral characterization of exoplanets and disks. The CAL2.0 will enable a new regime of highcontrast imaging, from reflected light imaging to young Myr old exoplanets in nearby star forming regions. The project will start early 2021, be developed alongside the GPI2.0 AO/IFS upgrades [38], and is expected to be installed in GPI late 2023. 


\section{Acknowledgment}

The authors thank the NRC Ideation Fund and CAL2.0 collaborators. We acknowledge the support of the Natural Sciences and Engineering Research Council of Canada (NSERC). The authors wish to recognize and acknowledge the very significant cultural role and reverence that the summit of Maunakea has always had within the indigenous Hawaiian community. We are most fortunate to have the opportunity to conduct observations from this mountain.

*christian.marois@nrc-cnrc.gc.ca; phone 1250 363-0023

\section{REFERENCES}

[1] Konopacky, Q. M., Barman, T. S., Macintosh, B. A., \& Marois, C., Science, 339, 1398 (2013).

[2] Keppler, M., Benisty, M., Müller, A., et al., A\&A, 617, A44 (2018).

[3] Nielsen, E. L., De Rosa, R. J., Macintosh, B., et al., AJ, 158, 13 (2019).

[4] Fernandes, R. B., Mulders, G. D., Pascucci, I., et al., ApJ, 874, 81 (2019).

[5] Marois, C., Doyon, R., Racine, R., \& Nadeau, D., PASP, 112, 91 (2000).

[6] Marois, C., Lafreni.re, D., Doyon, R., Macintosh, B., \& Nadeau, D., ApJ, 641, 556 (2006).

[7] Artigau, et al., Proc. SPIE, 10.1117/12.788390 (2008).

[8] Madurowicz, A., Macintosh, B., Chilcote, J., et al., JATIS, 5, 049003 (2019).

[9] Marois, C., Macintosh, B., Soummer, R., Poyneer, L., \& Bauman, B., in Proc. SPIE, 70151T (2008).

[10] Vigan, A., Moutou, C., Langlois, M., et al., MNRAS, 407, 71 (2010).

[11] Guyon, O. 2004, ApJ, 615, 562

[12] Bordé, P. J., \& Traub, W. A. 2006, ApJ, 638, 488

[13] Baudoz, P., Boccaletti, A., Baudrand, J., \& Rouan, D. 2006, in IAU Colloq. 200, 553-558

[14] Gerard, B. L., Marois, C., \& Galicher, R. 2018, AJ, 156, 106

[15] Macintosh, B., Graham, J. R., Ingraham, P., et al., PNAS, 111, 12661 (2014).

[16] Fitzsimmons et al., Proc. SPIE, 11448-262 (2020).

[17] Marois et al., Canadian Long Range Plan for A\&A White Papers, 59 (2019).

[18] Sozzetti, A., in Proc. SPIE, 104001E (2017).

[19] Gerard, B. L., Marois, C., Galicher, R., et al. 2019, in Proc. AO4ELT6

[20] Anglada-Escudé, G., Amado, P. J., Barnes, J., et al., Nature, 536, 437 (2016).

[21] Rajan, A., Rameau, J., De Rosa, R. J., et al., AJ, 154, 10 (2017).

[22] Samland, M., Molli.re, P., Bonnefoy, M., et al., A\&A, 603, A57 (2017).

[23] Nielsen, E. L., Rosa, R. J. D., Rameau, J., et al., AJ, 154, 218 (2017).

[24] Esposito, T. M., Kalas, P., Fitzgerald, M. P., et al., AJ, 160, 24 (2020).

[25] Dong, R., Zhu, Z., Rafikov, R. R., et al., ApJL, 809, L5 (2015).

[26] Keppler, M., Benisty, M., Müller, A., et al. 2018, A\&A, 617, A44

[27] Nielsen, E. L., De Rosa, R. J., Wang, J., et al., AJ, 152, 175 (2016).

[28] Lopez-Rodriguez, E., Alonso-Herrero, A., Diaz-Santos, T., et al., MNRAS, 478, 2350 (2018).

[29] Singh, G., Martinache, F., Baudoz, P., et al., PASP, 126, 586 (2014).

[30] Gerard, B. L., 2020b, PhD dissertation, https://dspace.library.uvic.ca/handle/1828/11755

[31] Gerard et al. 2020, JATIS, submitted

[32] Dunn, J., Kerley, D., Smith, M., et al., in Proc. SPIE, 1070317 (2018).

[33] Savransky, D., Thomas, S. J., Poyneer, L. A. et al., in Proc. SPIE, 914740 (2014).

[34] Give'On, A., Belikov, R., Shaklan, S., et al., Optics Express, 15, 12338 (2007).

[35] Mugnier, L. M., Blanc, A., Idier, J., Advances in Imaging and Electron Physics, 141, 1-76 (2006).

[36] Lamb, M. P., Correia, C., Sauvage, J.-F., et al., JATIS, 3, 039001 (2017).

[37] Lardière et al. 2020, Proc. SPIE, 11448-282

[38] Chilcote et al., Proc. SPIE, 11447-333 